\title{
Broad ligament myoma: a case managed by vaginal hysterectomy and broad ligament myomectomy
}

\author{
Prema Kania, Shubhdeep Kaur*
}

Department of Obstetrics and Gynaecology, Bombay Hospital Institute of Medical Sciences, Mumbai, Maharashtra, India

Received: 06 January 2022

Accepted: 01 February 2022

\section{*Correspondence:}

Dr. Shubhdeep Kaur,

E-mail: shubhdeepkaurdadiala@gmail.com

Copyright: $\odot$ the author(s), publisher and licensee Medip Academy. This is an open-access article distributed under the terms of the Creative Commons Attribution Non-Commercial License, which permits unrestricted non-commercial use, distribution, and reproduction in any medium, provided the original work is properly cited.

\begin{abstract}
The broad ligament is the commonest extra uterine site for leiomyoma but with low incidence rate. We present a patient with complaints of lower abdominal pain and premenstrual spotting per vaginum. Abdominal examination revealed a huge firm mass arising from the pelvis corresponding to 10-12-week size. Cervix was deviated to right and elevated but felt separable of the mass. Ultrasonographic examination showed left adnexal $9 \mathrm{~cm}$ fibroid beside the uterus suggesting broad ligament fibroid. Laparoscopy was planned but vaginal hysterectomy was attempted considering the expertise of the surgeon and previous 2 vaginal deliveries. Non descending vaginal hysterectomy was done to increase the space and working field for performing the broad ligament myomectomy. Extreme care was taken to avoid the ureteric injury. This case was reported because of the rare incidence of broad ligament leiomyoma and the difficulty in its operative management.
\end{abstract}

Keywords: Leiomyoma, Broad ligament leiomyoma, Ureteric injury, Vault suspension

\section{INTRODUCTION}

Leiomyoma is the most common tumor of the uterus. ${ }^{1}$ Broad ligament is the most common extra uterine site for the occurrence of leiomyoma; the incidence is $<1 \%{ }^{2,3}$ Other extra uterine sites of leiomyomas are the round ligament, ovarian ligament, and the ovaries. ${ }^{4}$ Leiomyoma in the broad ligament has been reported to reach a huge size which can mimic ovarian malignancy. ${ }^{5}$ These benign tumors in the broad ligament are usually asymptomatic but if neglected and reached an enormous size, it results in chronic pelvic pain, compression of the bladder and the bowel with dysfunction. It can lead to menstrual abnormalities with a coexisting intrauterine myoma.

\section{CASE REPORT}

A 40-years old multiparous female, P2L2, NVD, presented to a tertiary care center with complaints of abdominal pain with pelvic heaviness and premenstrual spotting. There were no disturbances in the bladder and bowel function. There were no other associated symptoms. General physical examination was fair. Her vitals were within normal limits. Her abdomen was distended with a huge pelvic-abdominal mass on left, approximately 8 centimeters in size. The mass was non-tender and firm. It has limited mobility from side to side. Pelvic examination revealed normal vulva and vagina; the cervix was drawn up and deviated to the right side; it was closed and grossly looking healthy. Abdominal ultrasound showed a huge solid mass measuring 9 centimeters in the left uterine adnexa. Her blood counts and serum biochemistry were within normal limits. Her tumor markers, that is, CA 125, CA 19-9 and CEA all were within normal limit, which were done to rule out any tube-ovarian cancerous pathology. USG KUB was done showing signs of mild hydronephrosis or hydroureter, probably secondary to the external compression of left lower ureter by a large 
adnexal fibroid. A diagnosis of broad ligament leiomyoma was suggested. She was scheduled for laparoscopic hysterectomy, but vaginal hysterectomy was done due to surgeon expertise and the patient having had 2 normal vaginal deliveries in the past.

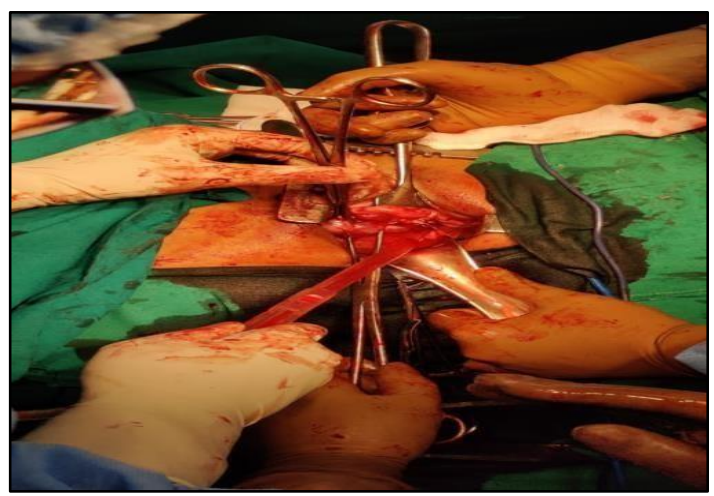

Figure 1: Left hydrosalpinx.

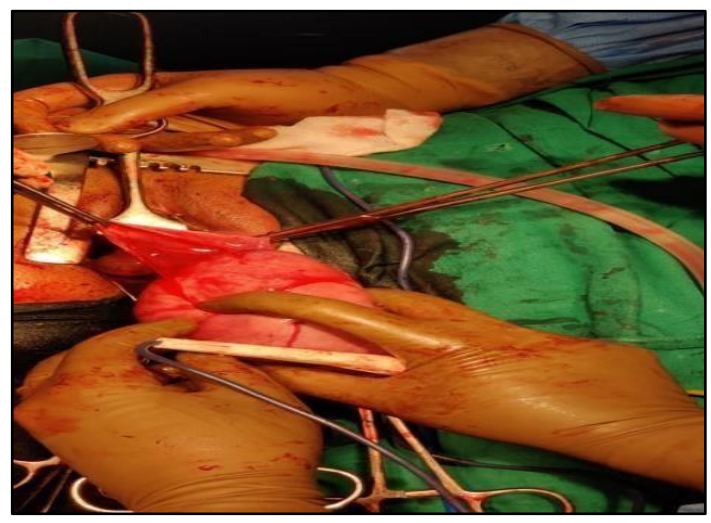

Figure 2: Broad ligament leiomyoma.

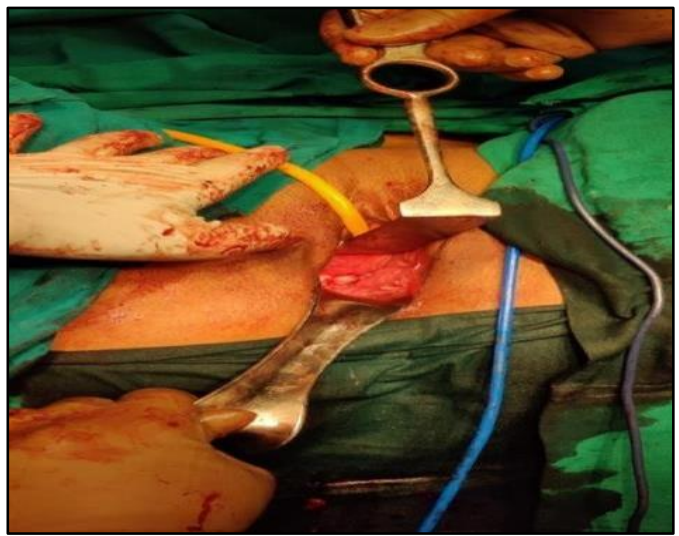

Figure 3: Vault closure done with vault suspension.

Intraoperatively, there was evidence of left hydro salpinx and decision was taken to do bilateral salpingectomy as well. Considering the young age of the patient and healthy ovaries, bilateral ovaries were preserved to avoid hormone replacement therapy. A huge left broad ligament leiomyoma measuring approximately 8/10 centimeters in its widest diameter, was felt. The round ligament stretched over the mass was incised by cautery and extending the incision down to the capsule of the mass. The plane of cleavage was dissected, and the mass was enucleated gently following the capsule, taking care at the bed of the myoma to avoid injury to the ureter. Maintaining intracapsular dissection of the myoma, close to the myoma and avoiding pulling it out sharply preventing injury to the ureter. The mass was separated easily. The mass was sent for histopathology measuring $4 \mathrm{kgs}$ and was confirmed to be a fibroid. The postoperative period of the patient was normal with discharge to home after 2 days.

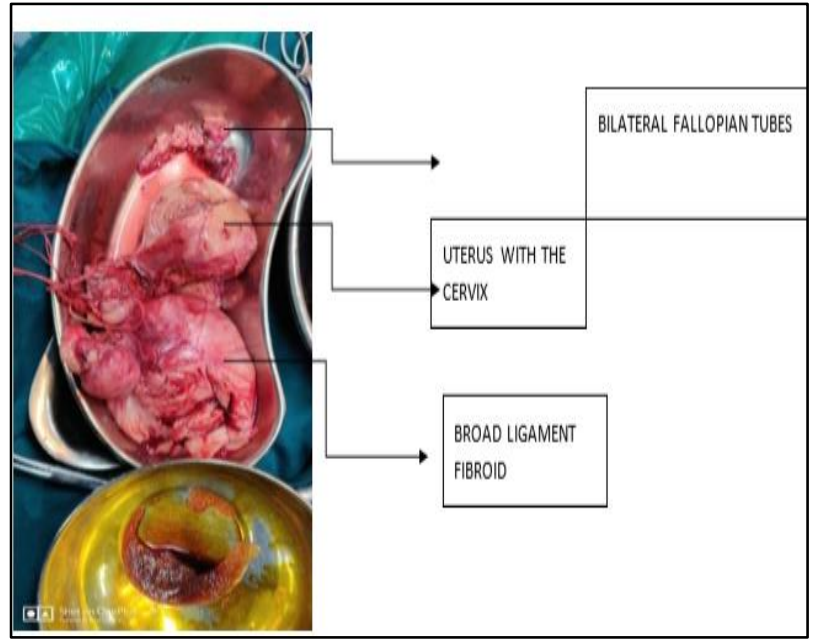

Figure 4: Post-procedure.

\section{DISCUSSION}

Broad ligament fibroid is a benign smooth muscle tumor which originates from the broad ligament hormone sensitive smooth muscle or secondarily from the uterine smooth muscle. ${ }^{5,6}$ Extra uterine leiomyoma which commonly occurs in the broad ligament are usually asymptomatic. Broad ligament fibroid has the potential to grow to a very large size. ${ }^{7}$ If reached an enormous size, it can present with pressure symptoms of pelvic pain and bladder and bowel dysfunction.

The case presented here had both pressure symptoms of chronic lower abdominal pain and heaviness in the lower abdomen. Enlargement of the leiomyoma can cause upward displacement of the uterus and it can become impacted in the pelvis leading to ureteric obstruction, urinary retention and or constipation. ${ }^{8}$

Diagnoses of broad ligament fibroid are always a challenge. The most useful modalities for detecting extrauterine leiomyomas are USG, CT, and magnetic resonance imaging (MRI). 9 "Bridging vessel sign" on imaging is helpful in the diagnosis of leiomyoma. ${ }^{10,11}$ Transvaginal ultrasound can diagnose broad ligament fibroid because it allows clear visual separation of the uterus and ovaries from the mass. Because of the location and size of broad ligament fibroids, surgery is challenging, especially since 
surrounding organs like ureters, intestines, and urinary bladder may be at risk.

\section{CONCLUSION}

We report broad ligament fibroid to emphasize the surgical complications they can pose. During surgery, one should be very careful about the ureteric course and surrounding organs. Non descending vaginal hysterectomy with bilateral salpingectomy was done to create space for performing the broad ligament myomectomy. We should keep dissection in the plane of cleavage intracapsular avoiding ureteric injury. Securing hemostasis at the bed of the myoma can be done by bilateral internal iliac ligation as the source of blood supply of the myoma might not be obvious.

Funding: No funding sources Conflict of interest: None declared

Ethical approval: Not required

\section{REFERENCES}

1. Neha G, Manisha L. A Rare Case of Giant Broad Ligament Fibroid with Cervical Fibroid Mimicking Ovarian Tumor. International Journal of Recent Trends in Science and Technology. 2014;10.2:208-9.

2. Bhatta N. Tumors of the Corpus Uteri. Jeffcoat's Principles of Gynecology. 6the Edition, Arnold Printers, London. 2001;470.

3. Parker WH. Uterine Myoma: An Overview of Development, Clinical Features and Management. . 2005;105.1:216-7.

4. Rajesh S, Adam M. Benign Metastasizing Leiomyoma: 'A Sheep in Wolf's Clothing. Community Oncology. 2013;10:122-5.

5. Dayananda KR, . Broad Ligament Fibroid Mimicking as Ovarian Tumor on Ultrasonography and CT Scan. Journal of Clinical Imaging Science. 2013;3.

Cite this article as: Kania P, Kaur S. Broad ligament myoma: a case managed by vaginal hysterectomy and broad ligament myomectomy. Int J Reprod Contracept Obstet Gynecol 2022;11:951-3. 